\title{
In utero fetal left ventricular rupture and pseudoaneurysm formation: a case report
}

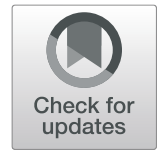

\author{
Sarah Heland ${ }^{1}$, Sarah Hope ${ }^{2}$, Andrew Edwards ${ }^{3}$, Rebecca Chalmers ${ }^{1}$, Alice Stewart ${ }^{4}$, Annie Kroushev',
} Bennett Sheridan ${ }^{5}$, Stuart Hooper ${ }^{6}$ and Kirsten R. Palmer ${ }^{7^{*}}$ (I)

\begin{abstract}
Background: Cardiac ventricular aneurysms affect 1 in 200,000 live births. To the best of our knowledge, no reported cases of a left ventricular pseudoaneurym and in utero rupture exist to guide optimal management.

Case presentation: We present a case of fetal left ventricular rupture with a large pericardial effusion, cardiac tamponade and subsequent pseudoaneurysm formation with concerns for a poor prognosis. Interventional drainage of the pericardial effusion led to resolution of tamponade and significant improvement in fetal condition. A multidisciplinary team was utilised to plan birth to minimise risk of pseudoaneurysmal rupture and a catastrophic bleed at birth.

Conclusion: For similar cases we recommend consideration of birth by caesarean section, delayed cord clamping and a prostaglandin E1 infusion, to reduce the systemic pressures on the left ventricle during transition from fetal to neonatal circulations, until definitive surgical repair. In this case, this resulted in a successful outcome.
\end{abstract}

Keywords: Cardiac pseudoaneurysm, Ventricular rupture, Interventional drainage, Perinatal cardiology

\section{Background}

Cardiac ventricular aneurysms affect 1 in 200,000 live births [1]. The first reported case diagnosed prenatally was published in 1990, however, to the best of our knowledge, no reported cases of a left ventricular pseudoaneurym and in utero rupture exist to guide optimal management [2].

\section{Case presentation}

A 25-year-old primiparous woman was referred for tertiary specialist fetal assessment at 23 weeks' gestation following a mid-trimester ultrasound indicating right outflow tract obstruction with pericardial effusion. Her pregnancy was previously uncomplicated. She had no significant past history. On review, a complex epicardial mass associated predominately with the left ventricle and a

\footnotetext{
* Correspondence: kirsten.palmer@monash.edu

${ }^{7}$ Department of Obstetrics and Gynaecology, Monash Health, and The Ritchie Centre, Hudson Institute of Medical Research, Clayton, Australia

Full list of author information is available at the end of the article
}

massive pericardial effusion was seen (Fig. 1), suggestive of an epicardial bleed. Remaining fetal anatomy was normal with no evidence of fetal hydrops or anaemia. An uncomplicated amniocentesis was performed and returned a normal Fluorescence In Situ Hybridization (FISH) and karyotype result; maternal infection screening was negative.

At $24+1$ weeks' gestation, the fetal pericardial effusion appeared stable but with an increasing epicardial thrombus. There was bidirectional flow between a pseudoaneurysmal defect and the left ventricle. An abnormal ductus venosus Doppler waveform, indicating poor ventricular function secondary to cardiac tamponade, and new onset ascites was evident.

Drainage of the massive pericardial effusion was deferred at 25 weeks' gestation, despite worsening hydropic features, due to bleeding into the pericardial space raising concerns for pseudoaneurysmal rupture (Fig. 2). At $26+1$ weeks' a pericardial drainage of $40 \mathrm{ml}$ of haemolysed blood-stained fluid was performed. Weekly follow

(c) The Author(s). 2021 Open Access This article is licensed under a Creative Commons Attribution 4.0 International License, which permits use, sharing, adaptation, distribution and reproduction in any medium or format, as long as you give appropriate credit to the original author(s) and the source, provide a link to the Creative Commons licence, and indicate if changes were made. The images or other third party material in this article are included in the article's Creative Commons licence, unless indicated otherwise in a credit line to the material. If material is not included in the article's Creative Commons licence and your intended use is not permitted by statutory regulation or exceeds the permitted use, you will need to obtain permission directly from the copyright holder. To view a copy of this licence, visit http://creativecommons.org/licenses/by/4.0/. The Creative Commons Public Domain Dedication waiver (http://creativecommons.org/publicdomain/zero/1.0/) applies to the data made available in this article, unless otherwise stated in a credit line to the data. 


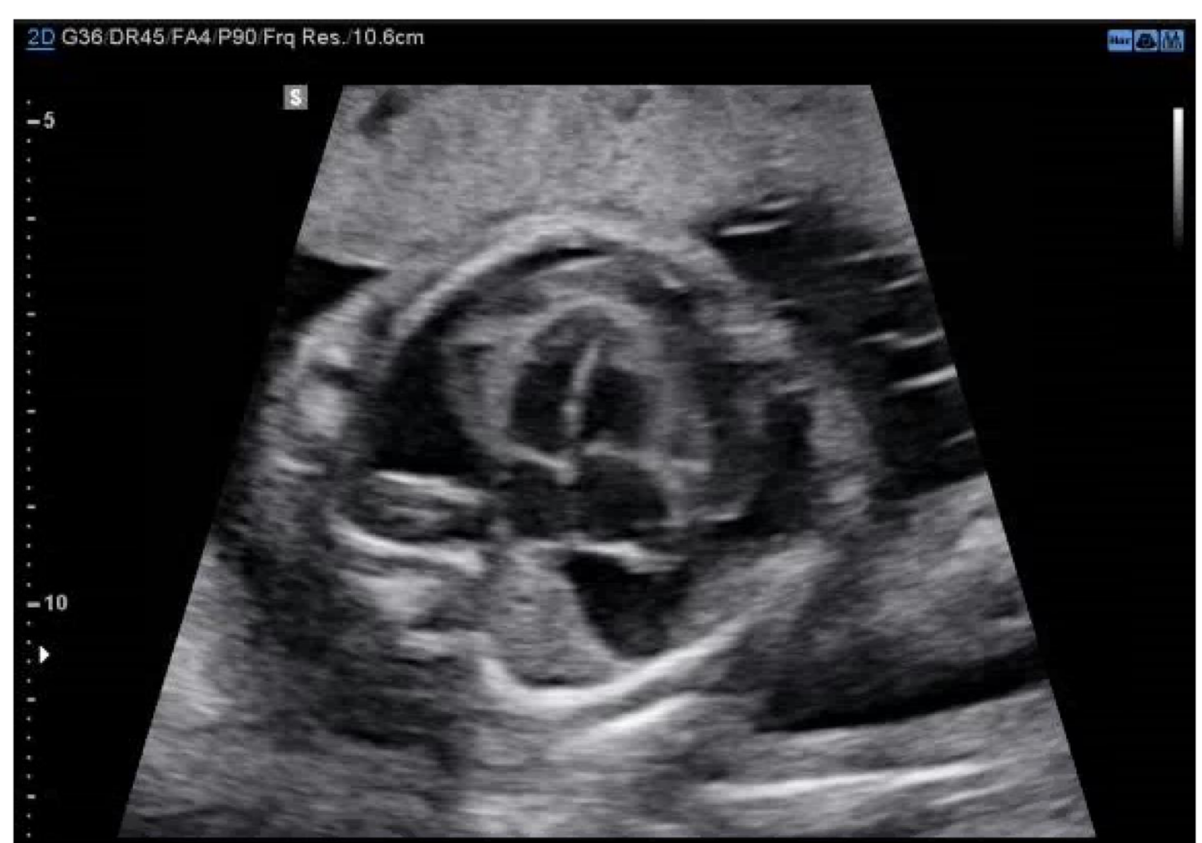

Fig. 1 Axial 4-chamber view of the fetal heart at $23+1$ weeks' gestation with a complex epicardial mass and a massive pericardial effusion seen

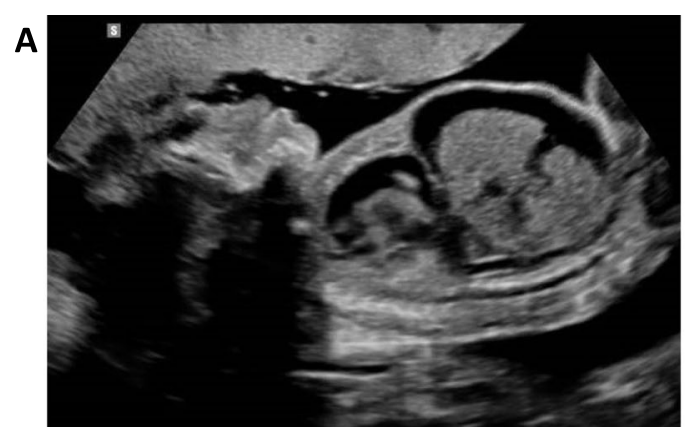

B

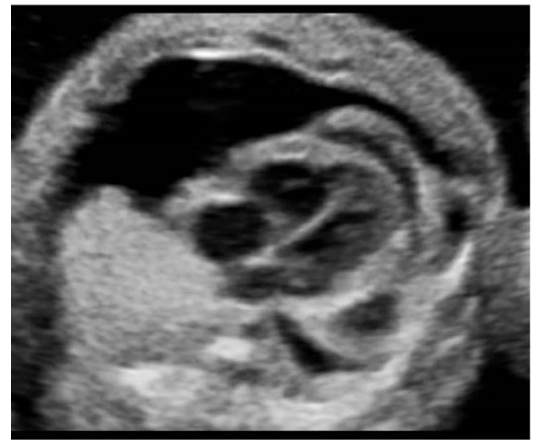

C

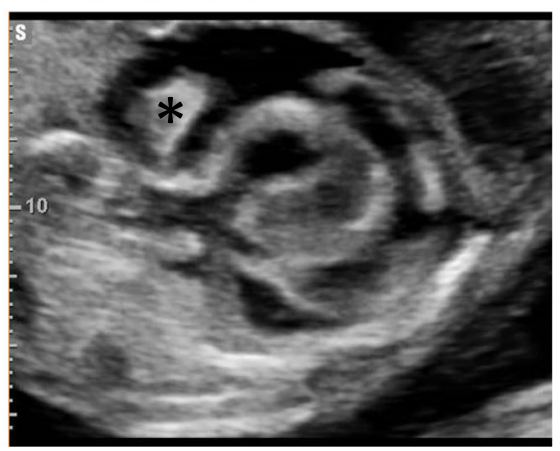

Fig. 2 Ultrasound assessment at 25 weeks' gestation, with evidence of fetal hydrops with ascites and a pleural effusion seen on sagittal view (a), a 4-chamber axial view of the heart shows persisting complex epicardial mass predominately associated with the left ventricle (b) and features suggestive of pseudoaneurysmal rupture with heterogenous material consistent with blood clot $\left(^{*}\right)$ seen in the pericardial effusion (c) 
up showed a small, gradually reducing, pericardial effusion, with resolution of the ascites and normalisation of the ductus venosus Doppler waveform.

A multidisciplinary team meeting with input from maternal fetal medicine, neonatology, paediatric cardiology, paediatric cardiac intensive care and perinatal physiology was conducted at 31 weeks' gestation to plan birth and postpartum care. Concerns existed of pseudoaneurysmal rupture and a potential catastrophic bleed during transition, as afterload pressures on the pseudoaneurysm increase due to increased systemic vascular resistance upon cord clamping and the loss of the low resistance placental circulation.

A caesarean birth was advised due to concerns of fetal stress and transient increases in placental resistance during labour increasing left ventricular afterload and the risk of pseudoaneurysmal rupture. Birth at term to minimise risks of prematurity and optimise birthweight prior to definitive surgical repair on cardiac bypass soon after birth was planned [3].

A fetal MRI showed normal neuroanatomy at 33 weeks' gestation. At $35+1$ weeks', a large left ventricular pseudoaneurysm persisted, but the pericardial effusion had completely resolved (Fig. 3).

An elective caesarean birth occurred at $37+5$ weeks' gestation following steroids to minimise the need for respiratory support. Cord clamping was deferred until after respiration was established to reduce pulmonary vascular resistance and allow left-to-right shunting through the ductus arteriosus to minimise the increase in left ventricular afterload associated with cord clamping. A female infant was born weighing $3209 \mathrm{~g}$ with Apgars of 9 at $1 \mathrm{~min}$ and 9 at $5 \mathrm{~min}$. In the immediate post-partum period, a prostaglandin E1 infusion was commenced to maintain ductal patency and reduce systemic and pulmonary vascular resistance. She remained stable and was transferred on day 1 of age to a quaternary paediatric cardiac centre for surgical management, undergoing aneurysmal resection with patch closure and ligation of a patent ductus arteriosus at 10 days of age. She made a good post-operative recovery and was discharged home at 19 days of age. On follow-up at 4 months of age she is thriving with a repeat echocardiogram showing good biventricular function.

\section{Discussion and conclusions}

Here we describe a case of fetal cardiac left ventricular pseudoaneurysm with in utero rupture that resulted in a successful outcome. Due to the small isolated defect, it is thought to have occurred secondary to a single coronary artery occlusion causing a localised myocardial infarction. Counselling the parents on the likelihood of having a live born infant and the subsequent prognosis following birth was challenging due to the rarity of the condition.

In view of the maternal primigravity and age, vaginal birth was considered to minimise maternal surgical morbidity in light of uncertain neonatal outcome. As labour and vaginal birth are known to cause transient episodes of fetal and placental hypoxia, leading to increased fetal acidosis and systemic arterial pressures, this mode of birth was thought to increase the possibility of pseudoaneurysmal rupture [4]. Utilising joint decision making with the parents, a caesarean birth was chosen as the optimal mode of delivery to minimise fetal risk.

Many key cardiovascular changes occur during transition from a fetal to neonatal circulation, including a significant reduction in the pulmonary vascular resistance,
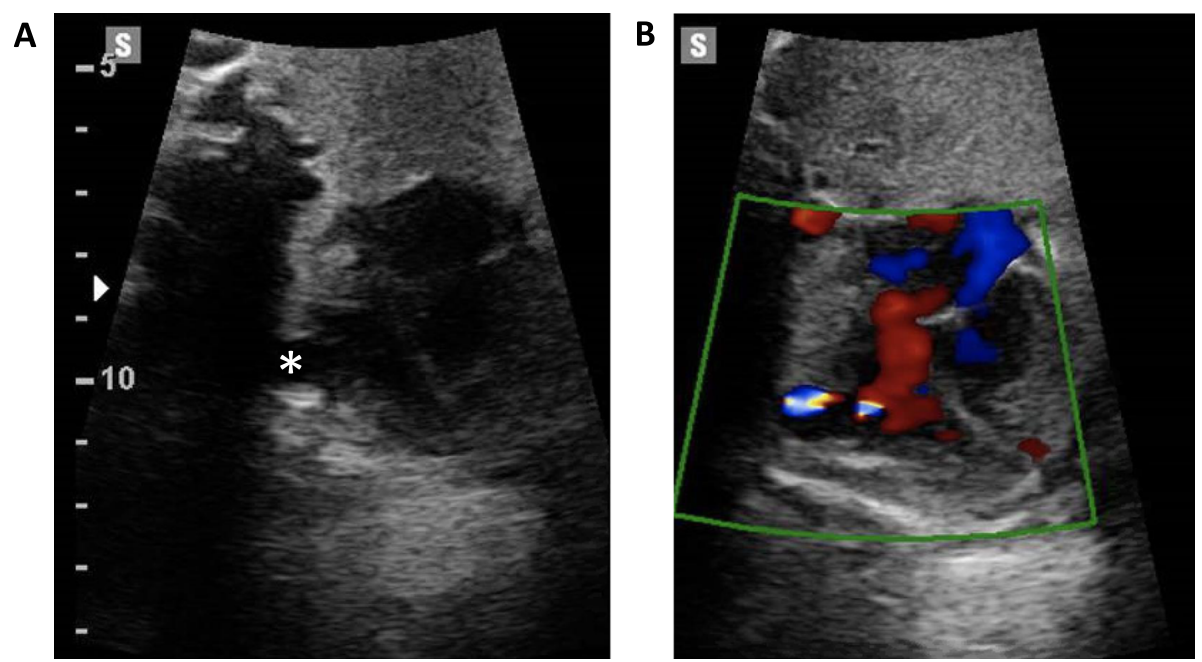

Fig. 3 Axial 4-chamber view of the fetal heart at $35+1$ weeks' gestation with a pseudoaneurysmal sac evident $\left(^{*}\right)$ arising from the left ventricle and resolution of the pericardial effusion (a). Blood flow into the pseudoaneurysm was clearly seen (b) 
an increase in systemic vascular resistance, and closure of the ductus arteriosus [5]. Prior to birth, left ventricular preload is predominately derived from umbilical venous return, while after birth it is from the pulmonary circulation [6]. Clamping of the umbilical cord removes the low resistance placental bed which significantly increases systemic vascular resistance and arterial blood pressure, such that in the first hour following birth the left ventricular cardiac output doubles [5, 6]. As the left ventricle transitions from pumping against the low resistance placental circulation to the high resistance neonatal systemic circulation, there is a significant increase in afterload, and in this case, an increased risk of pseudoaneurysmal rupture and catastrophic bleed [6]. To minimise this risk, umbilical cord clamping was delayed until neonatal respiration was well established, so that the pulmonary circulation could act as an alternate lower resistance pathway for left ventricular output, due to left-to-right ductal shunting. This prevents the rapid and large increase in left ventricular afterload associated with cord clamping [7]. The neonate was commenced on a prostaglandin E1 infusion to maintain a patent ductus arteriosus, minimizing systemic vascular resistance and decreasing left ventricular pressures [8].

We would recommend that in similar cases caesarean birth, deferred cord clamping and immediate commencement of a prostaglandin E1 infusion be considered until definitive surgical repair. Importantly, the possibility of an excellent outcome is also valuable in counselling and guiding families that may be impacted by this rare complication in the future.

\section{Abbreviations}

FISH: Fluorescence In Situ Hybridization; MRI: Magnetic Resonance Imaging

\section{Acknowledgements}

We thank the patient and their family in agreeing to share their experience to give guidance to others impacted by this rare condition.

\section{Authors' contributions}

SH1 and KP drafted the manuscript. AE, RC, SH2, BS, KP and AK made substantial contributions to the clinical management, acquisition and analysis of information. BS, AS and $\mathrm{SH} 3$ made substantial contributions to the interpretation of findings. All authors read and approved the final manuscript and agree to be accountable for the accuracy and integrity of the submitted work.

\section{Funding}

No funding was obtained for this paper.

\section{Availability of data and materials}

Data sharing is not applicable to this article as no datasets were generated or analysed during the current study.

\section{Declarations}

Ethics approval and consent to participate

Ethics approval was received from the Monash Health Research Office with HREC Reference Number: QA/66239/MonH-2020-220495(v1).

\section{Consent for publication}

Written consent was obtained from the patient involved for the use of their clinical information and images used in the case report.

\section{Competing interests}

The authors declare that they have no competing interests.

\section{Author details}

'Department of Obstetrics and Gynaecology, Monash Health, Clayton, VIC, Australia. ${ }^{2}$ Department of Paediatric Cardiology, Monash Heart, and Monash Cardiovascular Research Centre, Clayton, Australia. ${ }^{3}$ Department of Obstetrics and Gynaecology, Peninsula Health, and The Ritchie Centre, Hudson Institute of Medical Research, Clayton, Australia. ${ }^{4}$ Monash Newborn, Monash Health, Clayton, Australia. ${ }^{5}$ Cardiac Intensive Care, Royal Children's Hospital, Parkville, Australia. ${ }^{6}$ The Ritchie Centre, Hudson Institute of Medical Research, Clayton, Australia. ${ }^{7}$ Department of Obstetrics and Gynaecology, Monash Health, and The Ritchie Centre, Hudson Institute of Medical Research, Clayton, Australia.

Received: 30 December 2020 Accepted: 11 May 2021

Published online: 20 May 2021

References

1. Balakumar K. Prenatal diagnosis of left ventricular aneurysm. Indian J Radiol Imaging. 2009;19(1):84-6.

2. Gembruch U, Steil E, Redel DA, Hansmann M. Prenatal diagnosis of a left ventricular aneurysm. Prenat Diagn. 1990;10(3):203-9.

3. Steurer MA, Baer RJ, Keller RL, et al. Gestational age and outcomes in critical congenital heart disease. Pediatrics. 2017;140(4):e20170999.

4. Turner J, Mitchell MD, Kumar SS. The physiology of intrapartum fetal compromise at term. Am J Obstet Gynaecol. 2020;222(1):17-26.

5. Morton S, Brodsky D. Fetal physiology and the transition to Extrauterine life. Clin Perinatol. 2016;43(3):395-407.

6. Hooper SB, Binder-Heschl C, Polglase GR, et al. The timing of umbilical cord clamping at birth: physiological considerations. Matern Health Neonatol Perinatol. 2016:2:4

7. Bhatt S, Alison BJ, Wallace EM, et al. Delaying cord clamping until ventilation onset improves cardiovascular function at birth in preterm lambs. J Physiol. 2013;591:2113-26.

8. Akkinapally S, Hundalani SG, Kulkarni M, et al. Prostaglandin E1 for maintaining ductal patency in neonates with ductal-dependent cardiac lesions. Cochrane Database Syst Rev. 2018;2(2):CD011417.

\section{Publisher's Note}

Springer Nature remains neutral with regard to jurisdictional claims in published maps and institutional affiliations.

\footnotetext{
Ready to submit your research? Choose BMC and benefit from:

- fast, convenient online submission

- thorough peer review by experienced researchers in your field

- rapid publication on acceptance

- support for research data, including large and complex data types

- gold Open Access which fosters wider collaboration and increased citations

- maximum visibility for your research: over $100 \mathrm{M}$ website views per year

At BMC, research is always in progress.

Learn more biomedcentral.com/submissions
} 